for each individual. Based on the SOC, participants were categorised into different groups, consisting of pre-contemplation, contemplation, preparation, action, relapse, and maintenance. Adjusted odds ratios (AORs) and 95\% confidence intervals (CIs) were evaluated using binary logistic regression analyses.

Results Participants at the pre-contemplation stage were more likely to be older $(\mathrm{AOR}=1.07 ; 95 \% \mathrm{CI}=1.04-1.11)$, of female gender $(\mathrm{AOR}=1.54 ; 95 \% \mathrm{CI}=1.15-2.07)$, and have lower income $(\mathrm{AOR}=0.68 ; 95 \% \quad \mathrm{CI}=0.48-0.98)$ compared with those at contemplation, preparation or action stages. Relapse screeners were more likely to be older $(\mathrm{AOR}=1.08 ; 95 \%$ $\mathrm{CI}=1.03-1.13)$, at lower education level $(\mathrm{AOR}=0.54 ; 95 \%$ $\mathrm{CI}=0.35-0.82)$, and smokers $(\mathrm{AOR}=1.92 ; 95 \% \mathrm{CI}=1.09-$ 3.38) compared with maintenance screeners. The association was not found to be associated with marital status, occupation and self-perceived health status.

Conclusions The goal for promoting health programs is to facilitate forward movement from the pre-contemplation stage to contemplation, preparation, action and finally maintenance. This is one of the very few studies investigated factors associated with readiness to CRC screening by using the SOC. Our findings provide a foundation for developing interventions to improve long-term participation of CRC screening.

\section{IDDF2019-ABS-0255 ENDOSCOPIC RETROGRADE CHOLANGIOPANCREATOGRAPHY WITH BALLOON-ASSISTED ENTEROSCOPY IN PATIENTS WITH ROUX-EN-YANASTOMOSIS AND WHIPPLE OPERATION}

LiMin Zhang ${ }^{*}$, JiHeng Wang, YuQi He, DongLing Yu, Ge Gao. The Seventh Medical Center, Chinese PLA General Hospital, China

\subsection{6/gutjnl-2019-IDDFabstracts.213}

Background In patients with Roux-en-Y anastomosis(including hepaticojejunostomy and Roux-en-Y gastric bypass) and Whipple operation, endoscopic retrograde cholangiopancreatography (ERCP) can be challenging. We retrospective analyses our experience with ERCP using balloon-assisted enteroscopy (BAE) (BAE-ERCP) in patients with Roux-en-Y anastomosis and Whipple operation.

Methods ERCP was performed in 15 patients (4 pancreaticoduodenectomy and10 cholangiojejunostomy and 1Subtotal gastrectomy with Roux-en-Y reconstruction; age ranging from 4 to63 years) with balloon-assisted enteroscopy. Double- and single-balloon enteroscopy was applied in 5 and 10 patients, respectively.

Results Bile duct cannulation was successful in 13 of 15 cases $(86.7 \%)$, including simple stenosis of the anastomotic stoma $(n=2)$, intrahepatic bile duct stones $(n=10)$, and pancreatic cancer $(n=1)$. Cannulation failed because the guidewire could not pass through the anastomotic stenosis in one patient and because the endoscope could not enter the acute angle of the anastomosis of the afferent limb in the other patient. Adverse events included jaundice $(n=1)$ and perforation $(n=1)$, which were successfully treated by conservative therapy.

Conclusions ERCP with balloon-assisted enteroscopy in patients with Roux-en-Yanastomosis and Whipple operation is safe and useful but has unique complications. The success rate is lower than that of conventional ERCP.

\section{IDDF2019-ABS-0256 ASSESSMENT OF UPPER GASTROINTESTINAL SYMPTOMS IN PATIENTS WITH INFLAMMATORY BOWEL DISEASE}

Yasuhisa Sakata*, Nanae Tsuruoka, Motohiro Esaki. Saga University, Japan

\subsection{6/gutjnl-2019-IDDFabstracts.214}

Background We assessed the prevalence of upper GI symptoms in patients with IBD and examined the association between upper GI symptoms and disease manifestations, treatment, sleep problems, and fatigue.

Methods We investigated the upper GI symptoms using the frequency scale for symptoms of gastrointestinal reflux disease (FSSG) questionnaire comprising seven questions regarding reflux symptoms (RS) and five questions regarding acid-related dyspepsia (ARD). IBD outpatients completed questionnaires at their regular follow-up visit in our hospital. We also asked patients about sleep problems and fatigue with a questionnaire. We abstracted patients' data from medical records including age, sex, body mass index (BMI), smoking history, types of IBD, duration of IBD, surgical history, and medications.

Results Total 150 patients (83 male and 67 female), 105 Ulcerative Colitis (UC) and 45 Crohn's disease (CD) patients were included in the analysis. The mean age was $43.7 \pm 16.0$ years, mean BMI was $21.8 \pm 3.1$, and disease duration was 9.0 years. 30 patients $(20.0 \%)$ had a surgical history. Medications being used were 5-ASA in $136(90.7 \%)$, corticosteroid in $26(17.3 \%)$, immunomodulator in $54(36.0 \%)$, and biologics in $54(36.0 \%)$ patients. $62.2 \%$ of CD patients and $46.7 \%$ of UC patients reported upper GI symptoms. Female sex was associated with "heartburn after meals" (OR 3.09 (95\% CI 1.11-8.64), $\mathrm{p}=0.046)$. FSSG scores, RS scores and ARD scores were higher in CD patients than in UC patients $(\mathrm{p}=0.01, \mathrm{p}=0.03$, and $\mathrm{p}=0.01$. respectively). In particular, "unusual sensation in your throat" (OR 5.23 (95\% CI 1.2521.95), $\mathrm{p}=0.02$ ) and "burp a lot" (OR 2.71 (95\% CI 1.186.24), $\mathrm{p}=0.02$ ) were observed more frequently in $\mathrm{CD}$ patients. Upper GI symptoms were associated with sleep disturbance (OR 2.44 (95\% CI 1.12-5.34), $\mathrm{p}=0.025$ ), and fatigue (OR 3.20 (95\% CI 1.63-6.30), $\mathrm{p}=0.0009$ ).

Conclusions Upper GI symptoms in IBD patients were relatively common and have the potential to influence the sleep quality and feelings of fatigue. CD Patients suffer from upper GI symptoms more frequently compared to UC patients.

\section{IDDF2019-ABS-0261 BENEFICIAL EFFECTS OF CASSIA ANGUSTIFOLIA (SENNA LEAVES) TREATMENT IMPROVES CONSTIPATION, LIVER FUNCTIONS AND DEFENSE OF RED BLOOD CELLS IN TYPE 2 DIABETIC PATIENTS}

Rahul Kumar*, Bihari Gupta. Department of Pharmaceutical Sciences, Sardar Bhagwan Singh PG Institute of Biomedical Science and Research, Dehradun, India

\subsection{6/gutjnl-2019-IDDFabstracts.215}

Background Diabetes has been reported with constipation leading to the development of diabetic complications in spite of controlling the blood glucose levels by antidiabetic drugs. Our objective was to investigate the effects of Cassia Angustifolia (Senna leaves) extract on constipation, liver dysfunctions and defense of red blood cells in type 2 diabetic patients. 